\title{
Withdrawn notices
}

Sulaiman I, Mokoka MC, MacHale E, et al. P208 Behavioural feed-back education intervention to enhance adherence in patients with severe uncontrolled asthma, a randomised clinical trial. Thorax 2016;71:A198. doi:10.1136/thoraxjnl-2016-209333.351

This abstract has been withdrawn.

Thorax 2017;72:287. doi:10.1136/thoraxjnl-2016-209333.351wthn

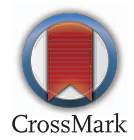

